\title{
Analysis of Fractional-order Linear Systems with Saturation Using Lyapunov's Second Method and Convex Optimization
}

\author{
Esmat Sadat Alaviyan Shahri Saeed Balochian \\ Department of Electrical Engineering, Islamic Azad University, Gonabad Branch Gonabad / Khorasan-e-Razavi 96916-29, Iran
}

\begin{abstract}
In this paper, local stability and performance analysis of fractional-order linear systems with saturating elements are shown, which lead to less conservative information and data on the region of stability and the disturbance rejection. Then, a standard performance analysis and global stability by using Lyapunov's second method are addressed, and the introduction of Lyapunov's function candidate whose sub-level set provide stability region and performance with a restricted state space origin is also addressed. The results include both single and multiple saturation elements and can be extended to fractional-order linear systems with any nonlinear elements and nonlinear noise that satisfy Lipschitz condition. A noticeable application of these techniques is analysis of control fractional-order linear systems with saturation control inputs.
\end{abstract}

Keywords: Fractional nonlinear systems, saturation systems, region of attraction, disturbance rejection, optimization problem.

\section{Introduction}

Recently, great attention has been paid to the analysis of fractional order control systems. There are attractive papers about stability and stabilization of fractional order systems including stability of linear fractional order system depending on location of system poles in the complex plane. In general, poles location analysis is a difficult task. Li et al. ${ }^{[1]}$ proposed the definition of Mittag-Leffler stability and introduced the fractional Lyapunov direct method (also called the Lyapunov's second method) that provides a way to analyze the stability of system without explicitly solving the differential equations. Lyapunov stability of the fractional differential equation was addressed and two approaches were presented: The direct one is intuitive but it leads to a large dimension parametric problem, while the indirect one is based on the continuous frequency distribution, leading to a parsimonious solution ${ }^{[2]}$.

An interesting application of stability analysis of the Lyapunov's theory is linear matrix inequalities (LMI) condition. This condition was investigated under commensurate order hypothesis ${ }^{[3]}$. A remarkable example demonstrated the advantage of fractional calculus in characterizing system behavior ${ }^{[4]}$. The stability of fractional-order nonlinear dynamic systems was studied using the Lyapunov direct method with the introduction of Mittag-Leffler stability and generalized Mittage-Leffler stability notion. With the proposed definitions, the decaying speed of the Lyapunov's function can be more generally characterized by including

Regular paper

Manuscript received September 5, 2013; accepted April 2, 2014

Recommended by Associate Editor Sheng Chen

(C) Institute of Automation, Chinese Academy of Science and Springer-Verlag Berlin Heidelberg 2015 the exponential stability and power-low stability. The decay of generalized energy of a dynamic system does not have to be exponential for the system to be stable ${ }^{[5]}$. This means that the energy decay actually could be of any rate, including power-low decay. The stability and stabilization problem of the fractional order linear system with nonlinear uncertain parameters was investigated and sufficient condition for the stability and stabilization of such a fractionalorder model were presented in terms of $\mathrm{LMI}^{[6]}$. Delavari et al. $^{[7]}$ studied stability analysis of fractional order nonlinear systems and proposed an extension of Lyapunov's second method for fractional-order systems using Bihari's and Bellmam-Gronwall's inequality. Also, Delavari et al. ${ }^{[8]}$ proposed a fractional order controller for nonlinear systems. In this method, the fractional controller converts the system with integer derivative into a system with desired fractional derivatives in order to increase the degree of freedom of stability. Extension of fractional order calculus in control is the enclosed polytopic system ${ }^{[9]}$, switched systems ${ }^{[10,11]}$ and nonstandard finite difference schemes for a fractionalorder Brusselator system ${ }^{[12]}$, etc. Additionally, the fractional calculus plays an important role in many chemical processes, physical phenomena, engineering systems, such as nanotechnology, viscoelastic material systems, quantum evolution of complex systems, robotics, signal processing and system identification, anomalous diffusion and wave propagation $^{[13-30]}$.

We mentioned some important papers about stability or application of fractional-order systems, but no paper has been addressed to analyze the fractional-order saturation systems. Because actuator saturation constitutes a fundamental limitation of many real systems and if ignored, it may cause undesired response or closed-loop instability, 
thus its study becomes necessary. Obviously, it is absolutely an open problem in the fractional calculus field.

Like most of the references, this paper has employed the Lyapunov's direct method for investigating stability. Furthermore, we know that stability analysis is not sufficient for programming and planning operation of nonlinear systems. And in many applications, computing the region of attraction and disturbance rejection is necessary. We will obtain stability conditions of fractional-order linear systems with actuator saturation and persistent disturbance. Then, we will find the region of attraction and disturbance rejection by using optimization based on the desired stability conditions. The proposed optimization problems give maximum volume invariant ellipsoid.

This paper is organized as follows. Information related to fractional-order derivative is given in Section 2. Problem statement is given in Section 3. Stability analysis and region of attraction in Section 4 and stability analysis and disturbance rejection in Section 5 are studied by Lyapunov's second method and optimization. Finally, the results are shown using an illustrative example.

\section{Information related to fractional- order derivative}

In this section, the main definitions concerning fractional's derivative are outlined.

Definition $\mathbf{1}^{[18,19,26]}$. (Reimann-Liouvill's fractional derivative). Reimann-Liouvill's fractional derivative of $q$ th order where $n-1<q<n$ is as

$$
{ }_{0} D_{t}^{q} f(t)=\frac{1}{\Gamma(n-q)}\left(\frac{\mathrm{d}}{\mathrm{d} t}\right)^{n} \int_{0}^{t} \frac{f(\tau)}{(t-\tau)^{q-n+1}} \mathrm{~d} \tau
$$

where $n$ is the first integer larger than $q$. Subscripts 0 and $t$ denote the two limits related to the operation of fractional derivation.

$$
\Gamma(q)=\int_{0}^{+\infty} \mathrm{e}^{-t} t^{q-1} \mathrm{~d} t
$$

where $\Gamma($.$) is the Gamma function generalizing factorial for$ non-integer arguments. It converges in the right half of complex plane.

Definition $\mathbf{2}^{[18,19,26]}$ (Caputo fractional derivative). Caputo fractional derivative of $q$-th order is

$$
{ }_{0}^{C} D_{t}^{q} f(t)=\frac{1}{\Gamma(n-q)} \int_{0}^{t} \frac{f^{(n)}(\tau)}{(t-\tau)^{q-n+1}} \mathrm{~d} \tau .
$$

It has been shown that there is a physical interpretation for initial values of Reimann-Liouvill fractional derivative and the interpretation of initial values of Caputo derivative is the same as in the classical integer-order case. We shall use Reimann-Liouvill definition throughout the paper.

Remark $\mathbf{1}^{[26]}$. In fractional order calculus, the rule for differentiating a product of two functions is

$$
D^{q}[f g]=\sum_{k=0}^{\infty}\left(\begin{array}{c}
q \\
k
\end{array}\right) D^{k}[g] \times D^{q-k}[f], q>0 .
$$

Remark $2^{[17]}$. The $q$-th order fractional derivative of function $g(x(t))=x^{2}(t)$ with respect to $t$ is given by

$$
{ }_{0} D_{t}^{q} g(x(t))=x(t) \times{ }_{0} D_{t}^{q} x(t)+L_{b}
$$

where

$$
L_{b}=\sum_{k=1}^{\infty} \frac{\Gamma(1+q)}{\Gamma(1+k) \Gamma(1-k-q)}{ }_{0} D_{t}^{k} x(t) \times{ }_{0} D_{t}^{q-k} x(t)
$$

with the following boundedness

$$
\left\|L_{b}\right\| \leq \beta\|x\|^{2}
$$

where $\beta$ is a positive real number.

By considering Remarks 1 and 2, the following remark can be concluded.

Remark 3. The $q$-th order fractional derivative of function $V(x(t), t)=x^{\mathrm{T}} P x$ with respect to $t$ is

$$
{ }_{0} D_{t}^{q} V(x, t)=x^{\mathrm{T}} P{ }_{0} D_{t}^{q} x(t)+L_{x}
$$

where $P$ is a positive definite matrix and

$$
L_{x}=\sum_{k=1}^{\infty} \frac{\Gamma(1+q)}{\Gamma(1+k) \Gamma(1-k-q)}{ }_{0} D_{t}^{k} x(t) P_{0} D_{t}^{q-k} x(t) .
$$

We could consider the following boundedness condition

$$
\left\|L_{x}\right\| \leq \delta\|x\|^{2}
$$

where $\delta=\beta \nu$ and $\nu$ is the largest of egenvalues of $P$.

\section{Problem statement}

Consider the fractional-order linear system with a decoupled block of saturator described by

$$
\begin{aligned}
& D_{t}^{q} x(t)=A x(t)+B \operatorname{sat}(u(t))+D w(t, x) \\
& u(t)=C x
\end{aligned}
$$

where $x \in \mathbf{R}^{n}$ is the state, $u \in \mathbf{R}^{m}$ is the control input, $w \in \mathbf{R}^{n_{w}}$ is function disturbance that is Lipschitz in $x$, and $A, B, D$ and $C$ are known real constant matrices of appropriate dimensions.

Remark $4^{[29-31]}$. Function $g: \aleph \rightarrow \mathbf{R}^{s}, \aleph \subseteq \mathbf{R}^{n}$, is said to be $L$-Lipschitz $L>0$, if

$$
|g(a)-g(b)|<L|a-b| .
$$

For any pair of points $a, b \in \aleph$, furthermore, we say that a function is Lipschitz if it is $L$-Lipschitz for some $L$.

$\operatorname{sat}(\cdot)$ denotes the normalized unit saturation function and defined by

$$
\begin{gathered}
\operatorname{sat}(C x)=\left[\operatorname{sat}\left(C_{i} x\right) \operatorname{sat}\left(C_{i} x\right) \cdots \operatorname{sat}\left(C_{i} x\right)\right] \\
i=1,2, \cdots, m \\
\operatorname{sat}\left(C_{i} x\right)=\left\{\begin{array}{cc}
1, & C_{i} x>1 \\
C_{i} x, & \left|C_{i} x\right| \leq 1 \\
-1, & C_{i} x<-1
\end{array}\right.
\end{gathered}
$$


Remark 5. The saturation function is Lipschitz in $x$ with Lipschitz constant $\lambda$, i.e.,

$$
\left\|\operatorname{sat}\left(x_{2}\right)-\operatorname{sat}\left(x_{1}\right)\right\| \leq \lambda\left\|x_{2}-x_{1}\right\| .
$$

Let $x$ lie in the region of

$$
\Re=\left\{x|| C_{i} x \mid \leq r_{i}\right\}, \quad i=1,2, \cdots, m, \quad r \in \mathbf{R}^{m} .
$$

If $r=1=\left[\begin{array}{llll}1 & 1 & \cdots & 1\end{array}\right]^{\mathrm{T}}$, the given system has a linear model, but we are interested in studying nonlinear models.

Definition $3^{[32-34]}$. If the initial state $x_{0}$ belongs to any set $D \subseteq \Re$ such that $\lim _{t \rightarrow \infty} x(t)=0$, with $w \equiv$ 0 , then $D$ will be called an $r$-level guaranteed region of attraction.

Definition $4^{[32-34]}$. If $x_{0} \equiv 0$ and for any number $\alpha$, $\|w\|_{2}^{2} \leq \alpha$, under the condition

$$
\lim _{t \rightarrow \infty} x(t)=0
$$

with $x(t) \in \mathbf{R}, \quad \forall t>0$, then we have $r$-level disturbance rejection bounded.

Our objectives are to find the largest number $\alpha$ and the largest region of attraction, $D$. In fact, we want to compute the $r$-levels:

1) $r$-level guaranteed region of attraction $D$;

2) $r$-level disturbance rejection.

Furthermore, we would like our objectives not to be conservative.

Remark $6^{[32]}$. For any candidate of Lyapunov's function as $V=x^{\mathrm{T}} P x$, where $P \in \mathbf{R}^{n \times n}$ is a positive definite matrix, $\xi_{P}(\alpha)=\{V<\alpha\}$ denotes $\alpha$-sublevel ellipsoid. It can be shown that volume of ellipsoid is proportional to $\alpha^{n} \operatorname{det}(P)$.

Lemma $1^{[32,33]}$. Let $V=x^{\mathrm{T}} P x, P=P^{\mathrm{T}}>0$ be Lyapunov's function, $F$ be a row vector in $\mathbf{R}^{n}$ and $c$ be a nonzero scalar. Then, the minimum of $\xi_{P}(\alpha)=\{V \leq \alpha\}$ along the hyper plane $\{x \mid F x=c\}$ has

$$
\alpha=\frac{c^{2}}{F P^{-1} F^{\mathrm{T}}} .
$$

Remark 7. For our objective, the necessary and sufficient condition for sublevel set $\xi_{P}(\alpha)$ containing in the region $\Re$ is

$$
\alpha=\min \frac{r_{i}^{2}}{C_{i} P^{-1} C_{i}^{\mathrm{T}}}
$$

where $C_{i}$ is the $i$-th row of $C$.

\section{Stability analysis and region of at- traction}

Our first concern is to find the stability condition and to compute the region of attraction when $w \equiv 0$.

Let the linear model be

$$
\Re=\{x \mid\|C x\| \leq 1\}
$$

and the nonlinear model

$$
\Re=\{x \mid\|C x\| \leq r\}, r>1 .
$$

They are studied separately.

Remark 8. For fractional-order systems, the exponential stability cannot be used to characterize the asymptotic stability, then a definition must be presented for fractionalorder systems.

Consider the following nonautonomous fractional-order system

$$
D_{t}^{q} x=F(x(t), t)
$$

where $F$ is a nonlinear function.

Definition $5^{[3]} \cdot t^{-\alpha}$-stability: Trajectory $x(t)=0$ of system (21) is $t^{-\alpha}$ asymptotically stable if the system is uniformly asymptotically stable and there are positive real $t_{0}, N$ and $\alpha$ such that

$$
\|x(t)\|<N\left(t-t_{0}\right)^{-\alpha}, \quad t>t_{0} .
$$

\subsection{Linear analysis}

Since system (11) never saturates in its region, the closedloop system behaves as

$$
D_{t}^{q} x(t)=(A+B C) x(t) .
$$

Theorem $\mathbf{1}^{[3]}$. The following system

$$
D_{t}^{q} x(t)=\hat{A} x
$$

is $t^{-\alpha}$ asymptotically stable if and only if there exists a positive definite matrix $P$ such that

$$
\left(-(-\hat{A})^{\frac{1}{2-q}}\right) P+P\left(-(-\hat{A})^{\frac{1}{2-q}}\right)<0
$$

where $(-\hat{A})^{\frac{1}{2-q}}$ is defined as $\mathrm{e}^{\frac{1}{2-q} \log (-\hat{A})}$.

Lemma 2. Fractional system (23) with order $0<q<1$ is asymptotically stable iff there exists a positive definite matrix $P$ such that

$$
\left(-(-(A+B C))^{\frac{1}{2-q}}\right)^{\mathrm{T}} P+P\left(-(-(A+B C))^{\frac{1}{2-q}}\right)<0 .
$$

Proof. System (23) is a fractional-order linear model, and by changing the following variable $A_{c l}=A+B C$, the system is converted to

$$
D_{t}^{q} x(t)=A_{c l} x(t) .
$$

Thus, for system (27),

$$
\left(-\left(-A_{c l}\right)^{\frac{1}{2-q}}\right) P+P\left(-\left(-A_{c l}\right)^{\frac{1}{2-q}}\right)<0 .
$$

Remark 9. This lemma provides the condition that the closed-loop system is in the stability region.

Optimization problem 1 (Region of attraction). For system (23), a 1-level region of attraction $D$ is given by the maximum volume invariant ellipsoid $\xi_{P}(1)$ containing in region $\Re$. 
We can compute it by solving the following convex optimization problem in variable $Q$, where $Q=P^{-1}$.

$$
\begin{array}{ll}
\max & \log \operatorname{det}(Q) \\
\text { s.t. } & \mathrm{C}_{i} Q C_{i}^{\mathrm{T}} \leq 1, \quad i=1,2, \cdots, m \\
& \mathrm{Q}>0 \\
& Q\left(-(-(A+B C))^{\frac{1}{2-q}}\right)^{\mathrm{T}}+\left(-(-(A+B C))^{\frac{1}{2-q}}\right) Q<0 .
\end{array}
$$

Then $\xi_{P}(1)$ is the largest invariant ellipsoid containing the region of attraction.

\subsection{Nonlinear analysis}

Since system (11) could be saturated in its region, the closed-loop system is in $\Re=\{x \mid\|C x\| \leq r\}$. It behaves like

$$
\begin{aligned}
& D_{t}^{q} x(t)=A x(t)+B \operatorname{sat}(C x) \\
& \quad x \in \Re \Leftrightarrow\left|C_{i} x\right| \leq r, \quad i=1,2, \cdots, m .
\end{aligned}
$$

By using the following theorem and lemma, we could obtain the first concern, i.e., the stability condition for system (30).

Theorem $2^{[1]}$ (The fractional Lyapunov's second method). Let $x=0$ be an equilibrium point of the following system

$$
D_{t}^{q} x(t)=g(x, t) .
$$

Let $V(x(t), t)$ be a candidate of the Lyapunov's function that is a continuously differentiable function and locally Lipschitz with respect to $x$, and $\beta_{i}(i=1,2,3)$ be Class_ $\kappa$ function satisfying

$$
\begin{gathered}
\beta_{1}(\|x\|) \leq V(x(t), t) \leq \beta_{2}(\|x\|) \\
D_{t}^{q} V(x(t), t) \leq-\beta_{3}(\|x\|)
\end{gathered}
$$

where $q \in(0,1)$, then $\lim _{t \rightarrow \infty} x(t)=0$.

Lemma $3^{[16,17]}$. Let $M$ and $N$ be real vectors of the same dimension. For any scalar $r>0$,

$$
M^{\mathrm{T}} N \leq r M^{\mathrm{T}} M+r^{-1} N^{\mathrm{T}} N .
$$

Theorem 3. System (30) is stable if and only if there are a symmetric positive define matrix $P>0$ and three scalars $r>0, \delta>0$ and $\lambda>0$ such that

$$
P A+r P B B^{\mathrm{T}} P+\left(r^{-1} \lambda^{2}+\delta\right) I<0
$$

where $\lambda$ is a Lipschitz constant and $I$ is an identical matrix with appropriate dimension. Also $\delta$ satisfies (10).

Proof. Consider the following Lyapunov's candidate that satisfies (32)

$$
V=x^{\mathrm{T}} P x .
$$

On the basis of Remark 3, we take the fractional derivative of (36)

$$
D_{t}^{q} V=x^{\mathrm{T}} P_{0} D_{t}^{q} x+L_{x}
$$

where

$$
L_{x}=\sum_{k=1}^{\infty} \frac{\Gamma(1+q)}{\Gamma(1+k) \Upsilon(1-k-q)}{ }_{0} D_{t}^{k} x(t) P_{0} D_{t}^{q-k} x(t) .
$$

According to (10),

$$
\left\|L_{x}\right\| \leq \delta\|x\|^{2} .
$$

By substituting (30) into (37),

$$
\begin{gathered}
{ }_{0} D_{t}^{q} V=x^{\mathrm{T}} P(A x+B \operatorname{sat}(C x))+L_{x}= \\
x^{\mathrm{T}} P A x+x^{\mathrm{T}} P B \operatorname{sat}(C x)+L_{x} .
\end{gathered}
$$

Applying Lemma 3,

$$
\begin{gathered}
x^{\mathrm{T}} P A x+x^{\mathrm{T}} P B \operatorname{sat}(C x)+L_{x} \leq \\
x^{\mathrm{T}} P A x+r x^{\mathrm{T}} P B B^{\mathrm{T}} P x+ \\
r^{-1}[\operatorname{sat}(C x)]^{\mathrm{T}}[\operatorname{sat}(C x)]+L_{x}
\end{gathered}
$$

and using (14) and (39),

$$
{ }_{0} D_{t}^{q} V \leq x^{\mathrm{T}}\left(P A+r P B B^{\mathrm{T}} P+\left(r^{-1} \lambda^{2}+\delta\right) I\right) x .
$$

According to (33), the system is stable when (35) holds. This theorem provides a stability condition for fractionalorder linear systems with any saturation actuator such as hyperbolic tangent sigmoid. Also, it applies to investigation stability condition for fractional-order systems nonlinear element that is Lipschitz in $x$.

Optimization problems 2 (Region of attraction). For system (30), on the basis of Remarks 6 and 7, the maximum volume invariant ellipsoid gives the $r$-level region of attraction $D$.

This could be computed by solving the following optimization problem in variable $P>0$.

$$
\begin{array}{ll}
\min & P \\
\text { s.t. } & C_{i} P^{-1} C_{i}^{\mathrm{T}} \leq r_{i}^{\mathrm{T}} \\
& P \geq 0 \\
& P A+r P B B^{\mathrm{T}} P+\left(r^{-1} \lambda^{2}+\delta\right) I<0 \\
& r>0, \delta>0 .
\end{array}
$$

By changing variable and cost function, this problem is converted into the convex optimization problem:

Optimization 3 (Region of attraction).

$$
\begin{array}{ll}
\max & \log \operatorname{det}(Q) \\
\text { s.t. } & \mathrm{C}_{i} Q C_{i}^{\mathrm{T}} \leq r_{i}^{\mathrm{T}} \\
& Q \geq 0 \\
& A Q+r B B^{\mathrm{T}}+\theta I \leq 0 \\
& \theta>0
\end{array}
$$

where

$$
\begin{aligned}
& \theta=\left(r^{-1} \lambda^{2}+\delta\right) \\
& Q=P^{-1}
\end{aligned}
$$


$\xi_{P}(1)$ is the largest region of attraction and contains the region $\Re$. We could use a cost function depending on the application. Trace $(Q)$ can be used instead of the maximized function that corresponds to maximizing the sum of the square of the major axes of $\xi_{P}(1)$.

\section{Stability analysis and disturbance re- jection}

Consider the closed-loop fractional-order system

$$
D_{t}^{q} x(t)=A x+B \operatorname{sat}(C x)+D w(t, x)
$$

where $w(t, x)$ is disturbance function that is Lipschitz in $x$, with the Lipschitz constant $L_{w}$ satisfies

$$
\left\|w\left(x_{1}, t\right)-w\left(x_{2}, t\right)\right\| \leq L_{w}\left\|x_{1}-x_{2}\right\| .
$$

We are interested in getting the stability condition of system (46). The following theorem and optimization problem are given for the desired stability condition and disturbance rejection for nonlinear saturation model and linear model consequently.

If the system could be saturated in its region, then we would consider (46) and study the stability condition and compute disturbance rejection by the following theorem and optimization problem.

Theorem 4. System (46) with $0<q<1$ is stable if and only if there are a symmetric positive definite matrix $P \geq 0$ and five scalars $s \geq 0, r \geq 0, \lambda>0, L_{w}>0$ and $\delta>0$ such that

$$
P A+r P B B^{\mathrm{T}} P+s P D D^{\mathrm{T}} P+\left(r^{-1} \lambda^{2}+s^{-1} L_{w}^{2}+\delta\right) I \leq 0
$$

where $\lambda>0$ and $L_{w}>0$ are the saturation Lipschitz constant and the disturbance Lipschitz constant, respectively, $s \geq 0$ and $r \geq 0$ are positive real numbers and $\delta>0$ satisfies (39). Also $I$ is an identical matrix with appropriate dimension.

Proof. Using the Lyapunov's candidate (36) and taking the fractional derivative of it and substituting (46), we get

$$
D_{t}^{q} V=x^{\mathrm{T}} P(A x+B \operatorname{sat}(C x)+D w)+L_{x}
$$

where (38) and (39) hold. We obtain

$$
D_{t}^{q} V=x^{\mathrm{T}} P A x+x^{\mathrm{T}} P B \operatorname{sat}(C x)+x^{\mathrm{T}} P D w+L_{x} .
$$

Considering Lemma 3, we have

$$
\begin{gathered}
D_{t}^{q} V \leq x^{\mathrm{T}} P A x+r x^{\mathrm{T}} P B B^{\mathrm{T}} P x+ \\
r^{-1}[\operatorname{sat}(C x)]^{\mathrm{T}}[\operatorname{sat}(C x)]+ \\
\quad s x^{\mathrm{T}} P D D^{\mathrm{T}} P x+s^{-1} w^{\mathrm{T}} w+L_{x} .
\end{gathered}
$$

Using inequalities (14), (39) and (47), we have

$$
\begin{aligned}
D_{t}^{q} V & \leq x^{\mathrm{T}}\left(P A+r P B B^{\mathrm{T}} P+r^{-1} \lambda^{2} I+\right. \\
& \left.s P D D^{\mathrm{T}} P+s^{-1} L_{w}^{2} I\right) x+L_{x} \leq \\
& x^{\mathrm{T}}\left(P A+r P B B^{\mathrm{T}} P+s P D D^{T} P+\right. \\
& \left.\left(r^{-1} \lambda^{2}+s^{-1} L_{w}^{2}+\delta\right) I\right) x .
\end{aligned}
$$

On the basis of (33), the system is stable iff (48) holds.

This theorem gives us a condition of stability for fractional-order linear systems with saturation control whenever there exists disturbance. Now, we obtain disturbance rejection of the system by the following optimization problem.

Optimization problem 4 (Disturbance rejection). For system (46), $\alpha$ is the largest $r$-level disturbance rejection by considering $\alpha=\frac{1}{t^{*}}$. Also, $t^{*}$ could be computed.

$\min t$

$$
\begin{array}{ll}
\text { s.t. } & P \geq 0, r>0, s>0, L_{w}>0, \lambda>0, \delta>0 \text { and } t>0 \\
& C_{i} P^{-1} C_{i}^{\mathrm{T}} \leq r_{i}^{\mathrm{T}} t \\
& P A+r P B B^{\mathrm{T}} P+s P D D^{\mathrm{T}} P+ \\
& \left(r^{-1} \lambda^{2}+s^{-1} L_{w}^{2}+\delta\right) I \leq 0 .
\end{array}
$$

By changing variables, this problem converts into the convex optimization problem.

Optimization problem 5 (Disturbance rejection).

$\min t$

$$
\begin{array}{ll}
\text { s.t. } & Q>0, r>0, s>0, \theta>0 \text { and } t>0 \\
& C_{i} Q C_{i}^{\mathrm{T}} \leq r_{i}^{\mathrm{T}} t \\
& A Q+r B B^{\mathrm{T}}+s D D^{\mathrm{T}}+\theta I \leq 0 \\
& Q=P^{-1} \text { and } \theta=r^{-1} \lambda^{2}+s^{-1} L_{w}^{2}+\delta .
\end{array}
$$

$\xi_{P}(\alpha)$ gives us the largest disturbance rejection and also $\xi_{P}(\alpha) \subseteq \Re$ whenever $x(0) \equiv 0$ and $\|w\|_{2}^{2} \leq \alpha$, then the trajectory of the system will never abandon $\xi_{P}(\alpha)$.

If the system never saturates in its region, the closed-loop system (46) behaves as

$$
D_{t}^{q} x(t)=(A+B C)+D w(t, x) .
$$

Lemma 4. System (55) with $0<q<1$ is stable if and only if there are a symmetric positive definite matrix $P \geq 0$ and two scalars $s \geq 0$ and $\delta>0$ such that

$$
P A_{c l}+s P D D^{\mathrm{T}} P+\left(s^{-1} L_{w}^{2}+\delta\right) I \leq 0
$$

where

$$
\mathrm{A}_{c l}=A+B C .
$$

Proof. Considering the Lyapunov candidate (36) and by taking fractional derivative of it and substituting (55), we get

$$
\begin{gathered}
D_{t}^{q} V=x^{\mathrm{T}} P\left(A_{c l} x+D w\right)+L_{x}= \\
x^{\mathrm{T}} P A_{c l} x+x^{\mathrm{T}} P D w+L_{x}
\end{gathered}
$$

where (38) and (39) hold.

By using Lemma 3, we have

$D_{t}^{q} V \leq x^{\mathrm{T}} P A_{c l} x+s x^{\mathrm{T}} P D D^{\mathrm{T}} P x+s^{-1} w^{\mathrm{T}} w+L_{x}$.

Using inequalities (39) and (47), we have

$$
\begin{gathered}
D_{t}^{q} V \leq x^{\mathrm{T}} P A_{c l} x+s x^{\mathrm{T}} P D D^{\mathrm{T}} P x+x^{\mathrm{T}}\left(s^{-1} L_{w}^{2}+\delta\right) x= \\
x^{\mathrm{T}}\left(P A+s P D D^{\mathrm{T}} P+\left(s^{-1} L_{w}^{2}+\delta\right) I\right) x .
\end{gathered}
$$


The system is stable iff (56) holds.

This lemma provides a stability condition for fractional linear systems whenever control is not saturated and there exists disturbance.

Optimization problem 6 (Disturbance rejection). For system (55), $\alpha$ is the largest 1-level disturbance rejection that could be computed.

$$
\begin{aligned}
& \alpha=\min \frac{1}{C_{i} P^{-1} C_{i}^{\mathrm{T}}}, \quad i=1,2, \cdots, m \\
& P>0, s>0, L_{w}>0 \text { and } \delta>0 \\
& P(A+B C)+s P D D^{\mathrm{T}} P+\left(s^{-1} L_{w}^{2}+\delta\right) I \leq 0 .
\end{aligned}
$$

Furthermore, this optimization problem can be converted into a convex optimization problem by changing variables as

$$
\begin{array}{ll}
\min & t \\
\text { s.t. } & Q>0, s>0 \text { and } \theta>0 \\
& C_{i} Q C_{i}^{\mathrm{T}}<t \\
& (A+B C) Q+s D D^{\mathrm{T}}+\theta I \leq 0
\end{array}
$$

where

$$
Q=P^{-1}, \quad t=\frac{1}{\alpha} \text { and } \theta=s^{-1} L_{w}^{2}+\delta .
$$

Also, $\xi_{P}(\alpha) \subseteq \Re$ whenever $x(0) \equiv 0$ and $\|w\|_{2}^{2} \leq \alpha$, then the trajectory of the system will never abandon $\xi_{P}(\alpha)$.

\section{Example}

We use an example to illustrate our results. The system is described by (11) with

$$
\begin{aligned}
& A=\left[\begin{array}{ll}
-20 & 1 \\
-10 & 0
\end{array}\right], B=\left[\begin{array}{c}
2 \\
-1
\end{array}\right] \\
& C=\left[\begin{array}{ll}
1 & 5
\end{array}\right], D=\left[\begin{array}{ll}
2 & 1
\end{array}\right] \\
& \text { with } w=\left[\begin{array}{c}
\sin \left(2 x_{1}\right) \\
\mathrm{e}^{-x_{1}}
\end{array}\right] \text { when } \Re=\{x|| C x \mid \leq 4\} .
\end{aligned}
$$

First, we compute the desired region of attraction for the given system. By solving (29) and (44), we get

$$
\mathrm{r}_{44}^{*}=0.277, \quad \theta_{44}^{*}=2.9301 \times 10^{-3}
$$

and the maximal ellipsoids are $\xi_{P^{*}}(1)$, where

$$
\begin{aligned}
& P_{44}^{*}=\left[\begin{array}{cc}
0.8407 & -0.0213 \\
-0.0213 & 8.9864
\end{array}\right] \\
& \mathrm{P}_{29}^{*}=\left[\begin{array}{cc}
3.7479 & 3.0569 \\
3.0569 & 26.3774
\end{array}\right] .
\end{aligned}
$$

Fig. 1 shows a 4-level region of attraction by the bold ellipsoid and 1-level region of attraction by the dot ellipsoid. To find the region of disturbance rejection when the system is saturated and when it is non-saturated, we solve (54) and (62). We get

$$
\begin{aligned}
& P_{54}^{*}=\left[\begin{array}{cc}
0.1629 & -0.2007 \\
-0.2007 & 1.7203
\end{array}\right] \times 10^{12} \\
& \alpha_{54}^{*}=1.1289 \times 10^{11} \\
& P_{62}^{*}=\left[\begin{array}{cc}
0.1031 & -0.5254 \\
-0.5254 & 7.1032
\end{array}\right] \times 10^{13} \\
& \alpha_{62}^{*}=2.6810 \times 10^{11}
\end{aligned}
$$

with

$$
\begin{aligned}
& r_{54}^{*}=2.5412 \times 10^{-12} \\
& s_{54}^{*}=3.5358 \times 10^{-13} \\
& \theta_{54}^{*}=1.9926 \times 10^{-12} \\
& s_{62}^{*}=7.5387 \times 10^{-14} \\
& \theta_{62}^{*}=4.9787 \times 10^{-13} .
\end{aligned}
$$

These give us the largest disturbance rejection when the system is saturated or not. If $\|w\|_{2}^{2} \leq \alpha, \alpha=\frac{1}{t^{*}}$, then the trajectory of the system will never abandon $\xi_{Q^{-1}}(\alpha)$. See the ellipsoid in Fig. 2. It shows the 4-level disturbance rejection by the bold ellipsoid and 1-level disturbance rejection by the dot curve.

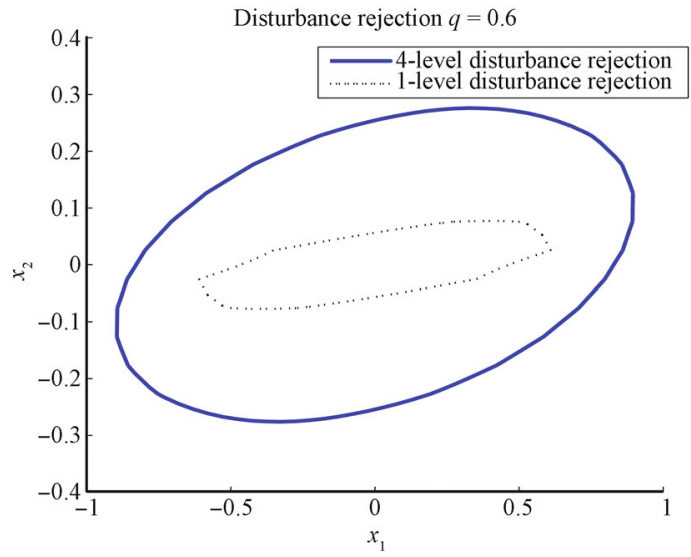

Fig. 1 Region of attraction

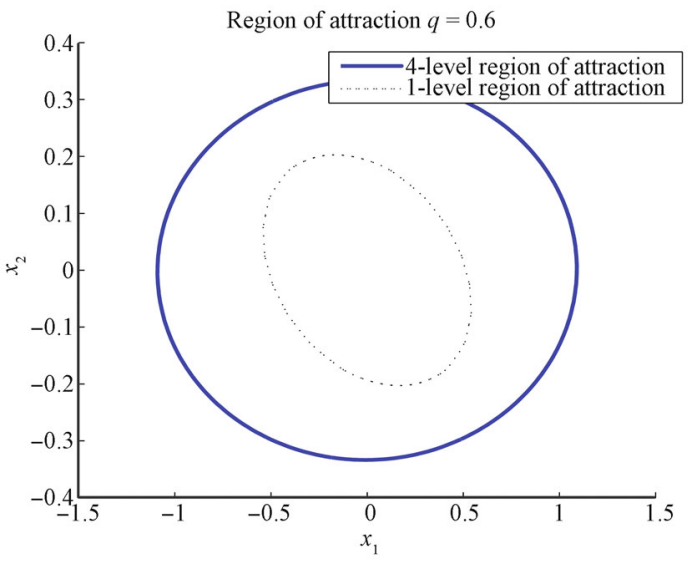

Fig. 2 Disturbance rejection 


\section{Conclusions}

In this paper, the Lyapunov's second method has been used for the fractional-order linear system with saturation to obtain the sufficient stability condition. A set of tools for performing local stability and performance analysis for the systems have been presented. This method could be used for fractional-order linear systems with any nonlinear elements that are Lipschitz in $x$. Our results are formulated in terms of LMI and lead to efficient computations. Also, the proposed LMI method is used for estimating the region of disturbance rejection of fractional order linear system with saturation element. Optimization problems are proposed for estimation of region of attraction and disturbance rejection. Finally, an illustrative example is shown for the effectiveness of the presented method when the given system is saturated or not.

\section{References}

[1] Y. Li, Y. Q. Chen, I. Podlubny. Mittag-leffler stability of fractional order nonlinear dynamic system. Automatica, vol. 45, no. 8, pp. 1965-1969, 2009.

[2] J. C. Trigeassou, N. Maamri, J. Sabatier, A. Oustalou. A Lyapunov approach to the stability of fractional differential equations. Signal Processing, vol. 91, no. 3, pp. 437-445, 2011.

[3] J. J. Sabatier, M. Moze, C. Farges. LMI stability conditions for fractional order systems. Computers and Mathematics with Applications, vol. 59, no. 5, pp. 1594-1609, 2010.

[4] Y. Li, Y. Q. Chen, I. Podlubny. Stability of fractional-order nonlinear dynamic systems: Lyapunov direct method and generalized Mittag-Leffler stability. Computers and Mathematics with Applications, vol.59, no.5, pp.1810-1821, 2010.

[5] Y. Q. Chen. Ubiquitous fractional order controls? In Proceedings of the Second IFAC Workshop on Fractional Derivatives and Applications, ISEP, Porto, Portugal, pp. 481-492, 2006.

[6] S. Y. Xing, J. G. Lu. Robust stability and stabilization of fractional-order linear systems with nonlinear uncertain parameters: An LMI approach. Chaos, Solitons and Fractals, vol. 42, no. 2, pp. 1163-1169, 2009.

[7] H. Delavari, D. Baleanu, J. Sadati. Stability analysis of caputo fractional-order nonlinear system revisited. Nonlinear Dynamics, vol.67, no. 4, pp. 2433-2439, 2012.

[8] H. Delavari, R. Ghaderi, A. Ranjbar, N. S. Momani. Fractional order controller for two-degree of freedom polar robot. In Proceedings of International Workshop on New Trends in Science and Technology, Ankara, Turkey, 2008.

[9] C. Farges, J. Sabatier, M. Moze. Fractional order polytopic systems: Robust stability and stabilisation. Advances in Difference Equations, 2011. (Online first)
[10] S. Balochian, A. K. Sedigh, A. Zare. Stabilization of multiinput hybrid fractional order systems with state delay. ISA Transactions, vol. 50, no. 1, pp. 21-27, 2011.

[11] S. Balochian, A. K. Sedigh. Sufficient condition for stabilization of linear time invariant fractional order switched systems and variable structure control stabilizers. ISA Transactions, vol. 51, no. 1, pp. 65-73, 2012.

[12] M. Y. Ongun, D. Arslan, R. Garrappa. Nonstandard finite difference schemes for a fractional order Brusselator system. Advance in Difference Equations, 2013. (Online first)

[13] H. S. Ahn, Y. Q. Chen. Necessary and sufficient stability condition of fractional-order interval linear systems. Automatica, vol. 44, no. 11, pp. 2985-2988, 2008.

[14] I. Petras. Fractional-order Nonlinear Systems Modeling, Berlin and Heidelberg, Germany: Springer-Verlag, 2011.

[15] M. O. Efe. Fractional fuzzy adaptive sliding-mode control of a 2-DOF direct drive robot arm. IEEE Transactions on Systems, Man, and Cybernetics, Part B: Cybernetics, vol. 38, no. 6, pp. 1561-1570, 2008.

[16] M. Pourgholi, V. J. Majd. A nonlinear adaptive resilient observer design for a class of lipschitz systems using LMI Journal of Circuits, Systems, and Signal Processing, vol. 30, no. 6, pp. 1401-1415, 2011.

[17] E. Amini Boroujeni, H. R. Momeni. Observer based control of a class of nonlinear fractional order system using LMI. World Academy of Science, Engineering and Technology, vol. 61, pp. 779-782, 2012.

[18] Y. Chen, B. M. Vinagre, D. Xue, V. Feliu. FractionalOrder Systems and Controls Fundamentals and Applications, London, UK: Springer-Verlag, 2010.

[19] I. Petras, D. Bednarova. Control of fractional-order nonlinear system: A review. Acta Mechanica et Automatica, vol. 5, no. 2, pp. 96-100, 2011.

[20] D. Baleanu, Z. B. Guven, J. A. T. Machado. New Trends in Nanotechnology and Fractional Calculus Applications, Netherlands: Springer, 2010.

[21] M. D. Ortigueira. An introduction to the fractional continuous-time linear systems: The 21st century systems. IEEE Circuits and Systems Magazine, vol. 8, no. 3, pp. 19$26,2008$.

[22] J. Sabatier, O. P. Agrawal, J. A. T. Machado. Advances in Fractional Calculus, Theoretical Developments and Applications in Physics and Engineering, London, UK: Springer, 2007.

[23] B. Guo, D. Huang. Existence and stability of standing waves for nonlinear fractional Schrödinger equations. Journal of Mathematical Physics, vol. 53, no. 8, Article number 083702, 2012.

[24] N. Laskin. Fractional quantum mechanics. Physical Review E, vol. 62, pp. 3135-3145, 2000. 
[25] R. Metzler, J. Klafter. The restaurant at the end of the random walk: Recent developments in the description of anomalous transport by fractional dynamics. Journal of Physics A: Mathematical and General, vol. 37, no.31, pp. R161-R208, 2004.

[26] S. Das. Functional Fractional Calculus, 2nd ed., Berlin Heidelberg, Germany: Springer-Verlag, pp. 1-220, 2011.

[27] L. Stamova, G. Stamov. Lipschitz stability criteria for functional differential systems of fractional. Journal of Mathematical Physics, vol. 54, no. 4, Article number 043502, 2013.

[28] R. Magin, M. D. Ortigueira, I. Podlubny, J. Trujillo. On the fractional signals and systems. Signal Processing, vol.91, no. 3, pp. 350-371, 2011.

[29] F. Liu, M. M. Meerschaert, S. Momani, N. N. Leonenko, W. Chen, O. P. Agrawal. Fractional differential equations. International Journal of Differential Equations, vol.2010, Article number 215856, 2010.

[30] L. G. Yuan, Q. G. Yang. Parameter identification and synchronization of fractional-order chaotic systems. Communications in Nonlinear Science and Numerical Simulation, vol. 17, no. 1, pp. 305-316, 2012.

[31] J. J. E. Slotin, W. A. Li. Applied Nonlinear Control, Englewood Cliffs, New Jersey, USA: Prentice Hall, 1991.

[32] H. Hindi, S. Boyd. Analysis of linear systems with saturation using convex optimization. In Proceedings of the 37th IEEE Conference on Decision and Control, IEEE, Florida, USA, pp. 903-908, 1998.

[33] L. Ghaoui, S. Niculescu. Advances in Linear Matrix Inequality Method in Control, Philadelphia, USA: Society for Industrial and Applied Mathematics, 2000.

[34] T. S. Hu, Z. L. Lin, B. M. Chen. An analysis and design method for linear system subject to actuator saturation and disturbance. Automatica, vol. 38, no. 2, pp. 351-359, 2002.

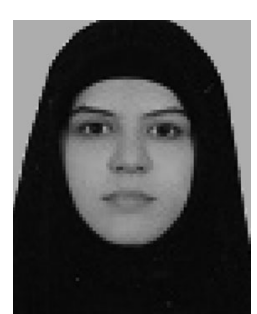

optimal control.

E-mail: ss_alariyan@yahoo.com

ORCID iD: 0000-0002-0138-3328

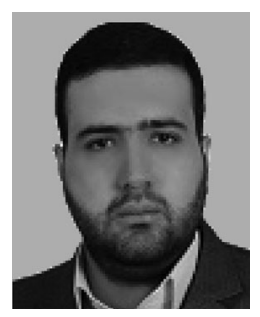

Saeed Balochian received the B. Sc. degree in communication system engineering and the M.Sc. degree in control and automation engineering in 2005 and 2007, and the $\mathrm{Ph}$. D. degree from the Islamic Azad University, science and research branch of Tehran. Currently, he is an assistant professor at Islamic Azad University of Gonabad branch, Iran.

His research interests include fuzzy systems and fractional derivative system control.

E-mail: Saeed.balochian@gmail.com (Corresponding author) ORCID iD: 0000-0003-3137-9167 